\title{
A Novel Sensor Based on Electropolymerization Poly(safranine) Film Electrode for Voltammetric Determination of 4-Nitrophenol
}

\author{
Xing-Yuan Liu \\ Department of Chemistry, Teachers' College of Simao, Yunnan 665000, China. 'E-mail: yunliuxy@sina.com.cn \\ Received September 8, 2009, Accepted February 26, 2010
}

\begin{abstract}
A novel poly(safranine)-modified electrode has been constructed for the determination of 4-nitrophenol (4-NP) in natural water sample. The electrochemical behavior of poly(safranine) film electrode and its electrocatalytic activity toward 4$\mathrm{NP}$ were studied in detail by cyclic voltammetry (CV) and adsorptive linear stripping voltammetry (LSV). All experimental parameters were optimized and LSV was proposed for its determination. In optimal working conditions, the reduction current of 4-NP at this poly(safranine)-modified electrode exhibited a good linear relationship with 4-NP concentration in the range of $8.0 \times 10^{-8}$ to $4.0 \times 10^{-5} \mathrm{~mol} \mathrm{~L}^{-1}$. The detection limit was $3.0 \times 10^{-8} \mathrm{~mol} \mathrm{~L}^{-1}$. The high sensitivity and selectivity of the sensor were demonstrated by its practical application for the determination of trace amounts of 4-NP in natural water and fruit samples.
\end{abstract}

Key Words: Sensor, Safranine, 4-Nitrophenol, Electropolymerization

\section{Introduction}

Phenol and substitute phenols have gained considerable attention in the past decade due to their toxic effects on human beings, animals and plants. Phenolic compounds are produced by a number of polluting processes, including industry-related methods of plastic, paint, antioxidant, petroleum, paper, drug and pesticide production. ${ }^{1}$ The compound 4-NP is known for its toxicity, carcinogenity and persistence in the environment. ${ }^{2-4}$ It has become a common pollutant in natural and wastewater. Moreover, 4-NP is involved in most of the degradation pathways of organophosphorous pesticides like fenitrothion, methyl-parathion, ethyl-parathion. These are decomposed in soil and water producing 4-NP as an intermediate or final product of the reaction. ${ }^{5,6}$ As a consequence, 4-NP is cited in the Environmental Protection Agency List of Priority Pollutants. ${ }^{7}$ Hence, the determination of 4-NP in water and food samples is of paramount importance. Obviously there is a strong interest for the development of simple, sensitive and reliable determination method.

The detection of 4-NP is usually accomplished using chromatographic techniques, such as gas chromatography, ${ }^{8,9}$ high performance liquid chromatography, ${ }^{10,11}$ liquid chromatography associated with mass spectroscopy, ${ }^{12}$ and capillary electrophoresis. ${ }^{13}$ Although sensitive and selective, these methods depend on multi-step sample clean-up procedures and are, therefore, generally time-consuming and require skilled personnel. Electrochemical method, which can offer fast, simple and direct real time analysis is one of the most used techniques in the determination of phenolic compounds. Electroanalytical methods have been proposed for 4-NP determination with a modified glassy carbon electrode(GCE), ${ }^{14-16}$ hanging mercury drop electrode (HMDE) $)^{17,18}$ and boron-doped diamond (BDD) electrode. The analytical signal is derived from the 4-electron reduction of the nitro group ${ }^{19-20}$ or by the direct 2-electron oxidation of phenol to the corresponding $o$-benzoquinone. ${ }^{21-23}$ The amount of 4-NP in seawater has been measured with differential pulse voltammetry. This is potentially useful for on-line monitoring. ${ }^{16}$
In recent years, water-soluble dyes, such as methylene blue, ${ }^{24}$ methylene green, ${ }^{25}$ carmine, ${ }^{26}$ and other dye derivatives, have been widely used as mediators to catalyze the reduction or oxidation of organic molecules. Safranine is a water-soluble red-colored phenazine-type dye used widely in paper and pharmaceutical industries. However, safranine polymer has rarely been used as a mediator to facilitate the electrochemical reaction of compounds.

In this work, a novel sensor for the detection of 4-NP was developed by the electropolymerization of safranine on the surface of a GCE. An electrochemical measurement was proposed for the direct determination of 4-NP in water and apple samples. The results were consistent with those of a standard chromatography procedure.

\section{Experimental Section}

Apparatus. All electrochemical experiments were carried out on a $\mathrm{CHI} 660 \mathrm{C}$ electrochemical workstation (CH Instrument Company, Shanghai, China) with a conventional three-electrode system. The working electrode was a GCE modified with electropolymerization of safranine. The auxiliary and reference electrodes were made of platinum wire and saturated calomel electrode (SCE), respectively.

Reagents. Analytical grade 4-NP was purchased from Shanghai Reagent Company (Shanghai, China). Stock standard solution was prepared by dissolving 4-NP in ethanol and then storing

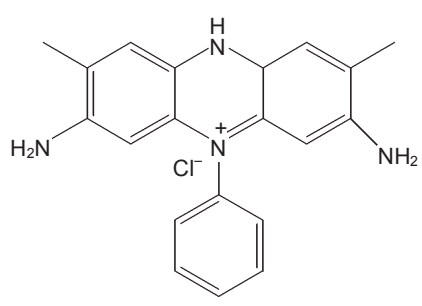

Figure 1. The molecular structure of safranine compounds. 
it in the refrigerator. An aqueous solution was prepared daily by simple dilution of the stock solution with $0.100 \mathrm{~mol} \mathrm{~L}^{-1}$ phosphate buffer solution (PBS, $\mathrm{pH}=6.0$ ). Safranine (Fig. 1) was obtained from Shanghai Reagent Company (Shanghai, China). All reagents are of analytical reagent grade unless stated otherwise. Double-distilled water is used throughout the experiment.

Preparation of the modified electrode. The GCE ( $3.0 \mathrm{~mm}$ in diameter) was polished to a mirror finish with polish paper and alumina slurry and cleaned consecutively and thoroughly in an ultrasonic cleaner with 1:1 $\mathrm{HNO}_{3}$, alcohol, and redistilled water. Electropolymerization of safranine on the GCE was accomplished by cyclic voltammetry(CV) in PBS ( $\mathrm{pH}$ 6.0) containing $2.0 \times 10^{-5} \mathrm{~mol} \mathrm{~L}^{-1}$ safranine. A poly(safranine) film was formed on the electrode surface by sweeps between -1.6 and $2.0 \mathrm{~V}$ for 15 cycles at a scan rate of $0.1 \mathrm{~V} \mathrm{~s}^{-1}$.

Sample preparation. A natural water sample was obtained from a dam located in Puer (Yunnan, China). The $\mathrm{pH}$ value was adjusted with $\mathrm{KH}_{2} \mathrm{PO}_{4}-\mathrm{Na}_{2} \mathrm{HPO}_{4}$. The apple sample was obtained from the local market. Its pretreatment followed the recommended process in literature. ${ }^{27}$ Briefly, the apple seedcase sample was mashed with a blender. Then, $25.0 \mathrm{~g}$ of pulp was mixed with $0.05 \mathrm{~L}$ acetonitrile and $1.5 \mathrm{~g} \mathrm{NaCl}$, and the organic phase was collected and evaporated on a steam bath. The final volume was adjusted to $0.005 \mathrm{~L}$ by the adding appropriate amount of dichloromethane. Part of the sample solution was transferred to PBS ( $\mathrm{pH}$ 6.0). It was then spiked with a known amount of 4-NP stock solution. This was used for the electrochemical determination.

Analytical procedure. An electrochemical cell containing $0.01 \mathrm{~L}$ supporting electrolyte $\left(0.1 \mathrm{~mol} \mathrm{~L}^{-1} \mathrm{PBS}, \mathrm{pH} 6.0\right)$ and a specific amount of standard 4-NP solution were used to perform electrochemical measurements. The solution was de-aerated with nitrogen for $10 \mathrm{~min}$. After the potential was held at $-0.7 \mathrm{~V}$ for $5 \mathrm{~s}$ in order to accumulate 4-NP on the modified electrode, $\mathrm{CV}$ or adsorptive linear stripping voltammetry(LSV) was performed and voltammograms were recorded. The reduction peak current, measured at approximately $-1.0 \mathrm{~V}$ in LSV, was applied for the electrochemical determination. After each measurement, the poly(safranine) film electrode was reactivated by successive cyclic potential sweeps between -1.2 and $+0.8 \mathrm{~V}$ at $0.1 \mathrm{~V} \mathrm{~s}^{-1}$ in PBS (pH 6.0). All experiments were carried out at room temperature.

\section{Results and Discussion}

Electrochemical behavior of 4-NP on the sensor. The redox behavior of 4-NP was studied using the poly(safranine)-modified electrode. Fig. 2 shows cyclic voltammograms of $4.0 \times 10^{-5}$ $\mathrm{mol} \mathrm{L}^{-1} 4-\mathrm{NP}$ on the sensor in PBS (pH 6.0). An obvious reduction peak $(\mathrm{Pc})$ appeared at about $-1.05 \mathrm{~V}$ during the cathodic sweep, and couple of not well-defined redox peaks ( $\mathrm{Pa} 1$ and $\mathrm{Pc} 1$ ) were observed in the potential range of -1.2 to $0.4 \mathrm{~V}$. The anodic peak potential (Epa1) and the cathodic potential (Epc1) were located at -0.28 and $-0.23 \mathrm{~V}$, respectively. The peak-topeak potential separation was about $0.05 \mathrm{~V}$, indicating that the 4-NP electroreduction was a reversible process. Its voltammetric behavior is consistent with the electrochemical characteristics of 4-NP reported elsewhere. ${ }^{14,15}$ Therefore, the same electron

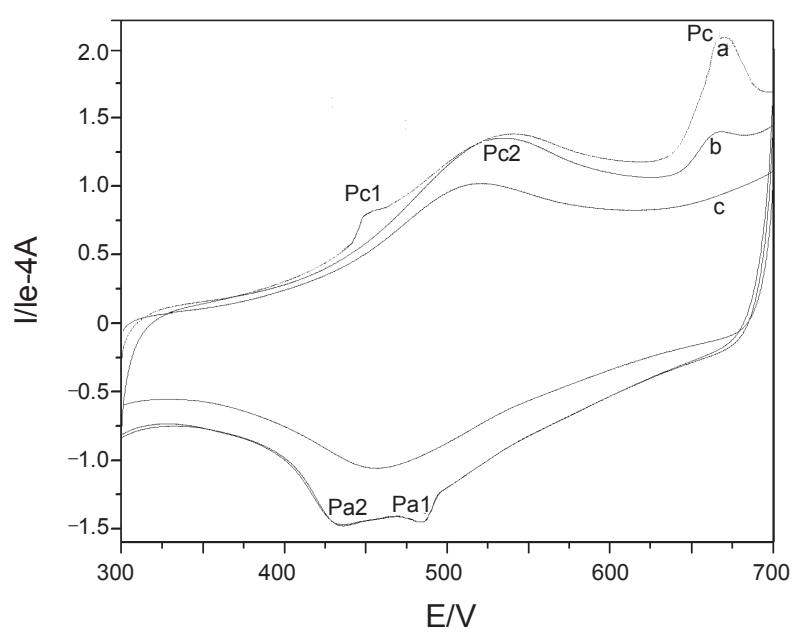

Figure 2. Voltammograms for a sensor in $0.100 \mathrm{~mol} \mathrm{~L}^{-1} \mathrm{pH} 6.0$ PBS with (curve a and b) and without (curve c) $4.0 \times 10^{-5} \mathrm{~mol} \mathrm{~L}^{-1} 4-\mathrm{NP}$. Curve $\mathrm{a}$ and $\mathrm{b}$ represent the first and the second cycle respectively. Scan rate: $100 \mathrm{mV} \mathrm{s}^{-1}$.

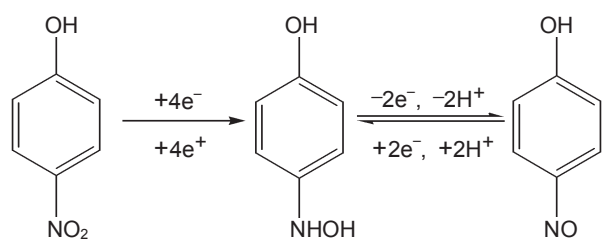

Scheme 1. Molecular structures and electrochemical reaction mechanism of 4-nitrophenol on the sensor.

transfer mechanism of 4-NP at the poly(safranine) film electrode can be applied in this study (Scheme 1).

The Pc peak was the result of an initial $4 \mathrm{e}^{-} / 4 \mathrm{H}^{+}$reduction of the nitro group to the hydroxylamine species. The couple Pa1/ Pc1 represented for the oxidation of hydroxylamine species and the reduction of the NO group, each transferring $2 \mathrm{e}^{-}$and $2 \mathrm{H}^{+}$. Moreover, the significant decrease of the current peak of the Pc process in the second scan (Fig. 2) could be related to the adsorption of 4-NP on the electrode surface. During the first cycle, most of the 4-NP adsorbed was reduced, and the second reduction peak appeared smaller than it was previously.

With the voltammogram of the sensor in $0.100 \mathrm{~mol} \mathrm{~L}^{-1} \mathrm{PBS}$ (pH 6.0) and in the absence of 4-NP (curve c) as basis, another couple of redox peaks ( $\mathrm{Pa} 2$ and $\mathrm{Pc} 2$ ) in curve $\mathrm{b}$ was ascribed to the redox reaction of the safranine polymer film. However, the current of curve $b$ was different from that of curve $c$ around $-0.4 \mathrm{~V}$ region. The current difference could be caused by the adsorption of an unstable intermediate and/or the adsorption of the final product of the electrode reaction (Scheme 1).

Furthermore, adsorptive linear stripping voltammetry was applied to study the electrochemical behavior of 4-NP on the sensor. Fig. 3 shows the adsorptive linear stripping voltammograms of bare GCE (curve c) and the sensor with (curve a) and without (curve b) $4.0 \times 10^{-6} \mathrm{~mol} \mathrm{~L}^{-1} 4-\mathrm{NP}$ in PBS ( $\mathrm{pH}$ 6.0). One reduction peak of safranine polymer film was also observed at around $-0.50 \mathrm{~V}$ on the sensor (curve $\mathrm{a}$ and $\mathrm{b}$ ). The voltammetric behavior of 4-NP on a bare GCE was also investigated in 


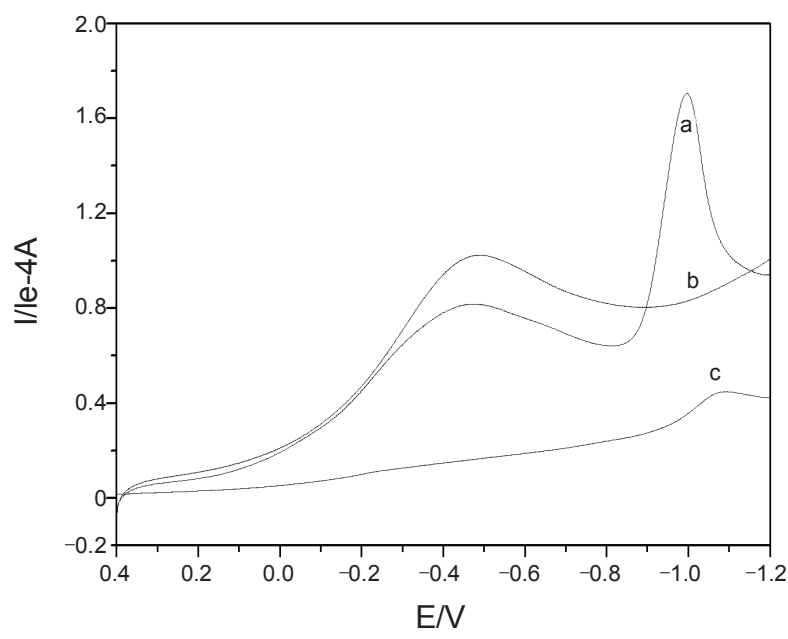

Figure 3. Adsorptive linear stripping voltammograms for $4.0 \times 10^{-6} \mathrm{~mol}$ $\mathrm{L}^{-1} 4-\mathrm{NP}$ on the sensor (a) and a bare glassy carbon electrode (c). Curve b represents linear sweep voltammogram of the sensor in $0.100 \mathrm{~mol} \mathrm{~L}^{-1}$ $\mathrm{pH}$ 6.0 PBS in the absence of 4-NP. Scan rate: $100 \mathrm{mV} \mathrm{s}^{-1}$.

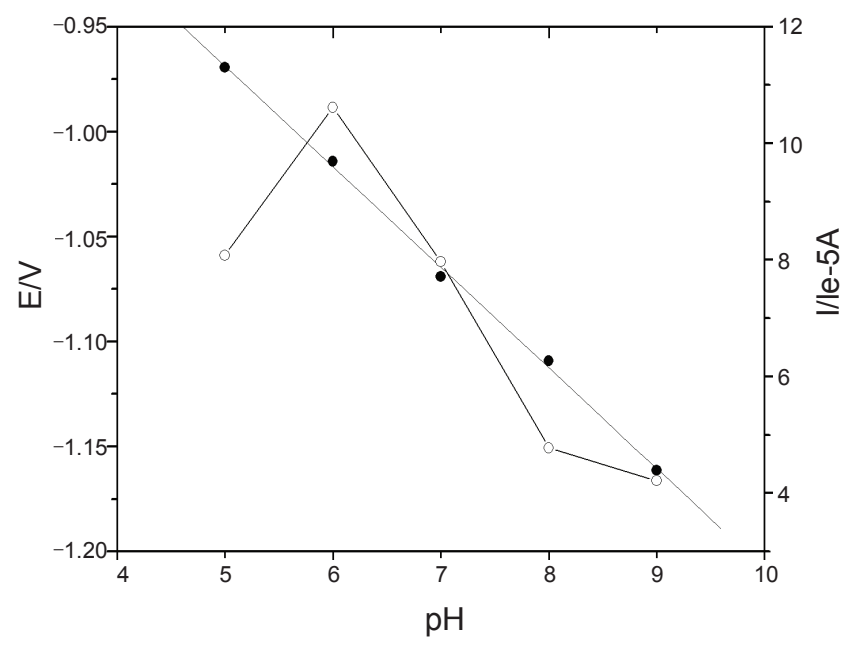

Figure 4. Effect of $\mathrm{pH}$ on the peak potential (full circle) and current (open circle) of $4.0 \times 10^{-6} \mathrm{~mol} \mathrm{~L}^{-1} 4-\mathrm{NP}$ on the sensor. Supporting electrolyte: $1 / 15 \mathrm{~mol} \mathrm{~L}^{-1}$ phosphate buffer.

$0.100 \mathrm{~mol} \mathrm{~L}^{-1}$ PBS. Only a small peak was observed at -1.08 $\mathrm{V}$ when the 4-NP concentration was controlled at $4.0 \times 10^{-6} \mathrm{~mol}$ $\mathrm{L}^{-1}$. However, the reduction peak current of 4-NP increased significantly, and the peak potential shifted positively to -1.0 V when the poly (safranine) film-modified GCE was employed. The remarkable peak current enhancement was undoubtedly attributed to the extraordinary properties of the polymer film, such as its three-dimensional distribution mediators and strong adsorption ability. In Fig. 1, phenazine groups are present in the chemical structure of safranine, Thus, these are also present in the structure of polymer (safranine). Moreover, a phenyl group is present in the structure of 4-NP. Therefore, during the process of pre-concentration, 4-NP can be accumulated in the surface of the electrode via " $\pi-\pi$ " hydrophobic interaction and/or hydrogen bonding with the poly (safranine) film to enhance the current response. These results suggest that the sensor exhibits

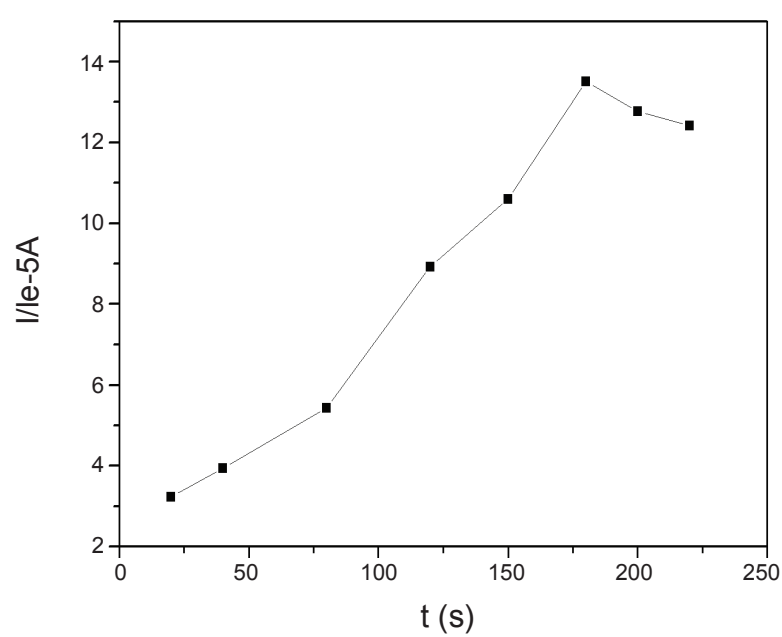

Figure 5. Influence of accumulation time on the reduction peak current of $4.0 \times 10^{-6} \mathrm{~mol} \mathrm{~L}^{-1} 4-\mathrm{NP}$ in $0.100 \mathrm{~mol} \mathrm{~L}^{-1} \mathrm{pH} 6.0 \mathrm{PBS}$. Accumulation potential: $-0.70 \mathrm{~V}$.

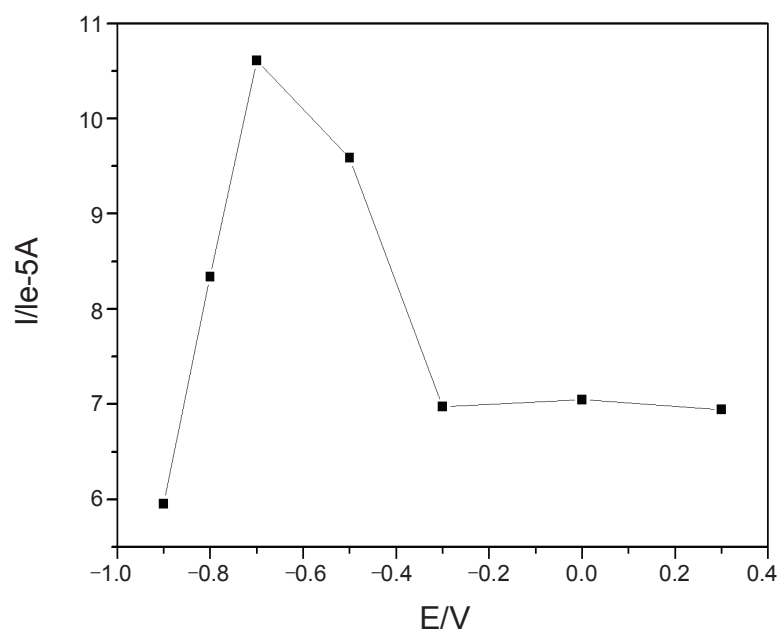

Figure 6. Effect of the accumulation potential on the reduction peak current of $4.0 \times 10^{-6} \mathrm{~mol} \mathrm{~L}^{-1} 4-\mathrm{NP}$ in $0.100 \mathrm{~mol} \mathrm{~L}^{-1} \mathrm{pH}$ 6.0 PBS. Accumulation time: $180 \mathrm{~s}$.

strong adsorptive ability and subtle electronic properties for 4-NP.

Influence of solution $\mathrm{pH}$. The effect of $\mathrm{pH}$ on the peak current and peak potential of 4-NP is displayed in Fig. 4. The reduction peak potential shifted negatively with increasing $\mathrm{pH}$ values. In the $\mathrm{pH}$ range of 5.0 - 9.0, a linear relationship existed between them. The regression equation was: $\mathrm{Epc}=-0.048 \mathrm{pH}-0.7294$ $(\mathrm{r}=0.998)$. A slope of approximately $0.05 \mathrm{~V} / \mathrm{pH}$ suggests that the overall process was proton-dependent and that the electron transfer step was preceded by protonation, with an equal number of protons and electrons involved in the 4-NP reduction. On the other hand, the reduction peak current $v s$. $\mathrm{pH}$ dependence revealed that the peak current had a maximum value at $\mathrm{pH}$ 6.0. The current decreased sharply in both directions for higher and lower $\mathrm{pH}$ values. For this reason, all subsequent experiments using the sensor were carried out in phosphate buffer at $\mathrm{pH}$ 6.0. 


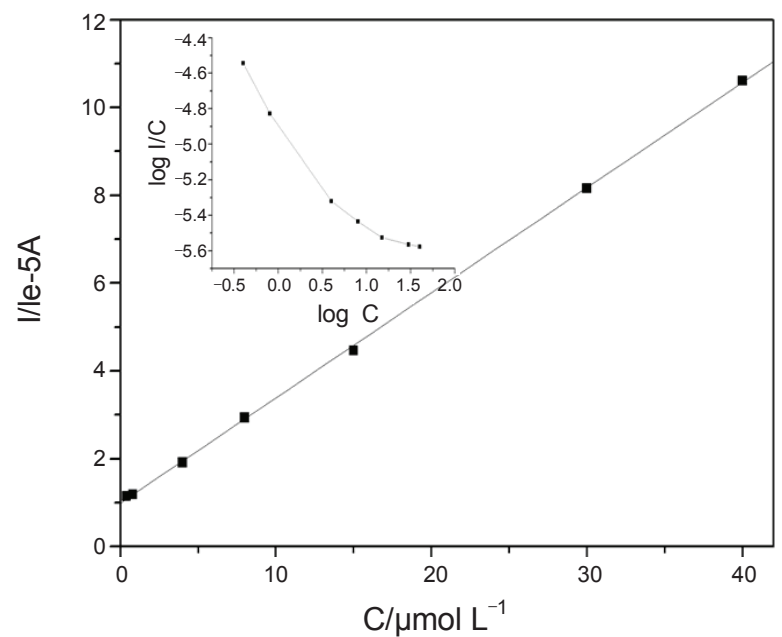

Figure 7. Calibration curve for the determination of 4-NP on the sensor. Inset: The $\log \mathrm{i} / \mathrm{C} v s . \log \mathrm{C}$ plot.

Influence of accumulation time and accumulation potential. Effects of accumulation time on the reduction peak current of $4.0 \times 10^{-6} \mathrm{~mol} \mathrm{~L}^{-1} 4-\mathrm{NP}$ were investigated by LSV, and the results are illustrated in Fig. 5. The peak current increased with the accumulation time in the range of $20-180 \mathrm{~s}$. This indicated the occurrence of adsorptive accumulation of 4-NP on the surface of the modified electrode. However, further increase in accumulation time did not increase the amount of 4-NP at the electrode surface owing to surface saturation. Therefore, $180 \mathrm{~s}$ was selected as optimum accumulation time for the determination of 4-NP on the sensor.

Fig. 6 shows the effect of accumulation potential on the response of 4-NP. A maximum current response was reached at the accumulation potential value of $-0.7 \mathrm{~V}$, and a sharp decrease in current was observed in both sides of this potential. Thus, an accumulation potential of $-0.7 \mathrm{~V}$ was used in subsequent studies to achieve optimum results for 4-NP determination.

Influence of scan rate. The effect of scan rate on the reduction of 4-NP was investigated by adsorptive linear stripping voltammetry in the range of $0.025-0.2 \mathrm{~V} \mathrm{~s}^{-1}$. A good linear relationship between the peak current and scan rate was observed in the range studied. The regression equation was: Ipc $(10 \mu \mathrm{A})=0.055 v$ $\left(\mathrm{mV} \mathrm{s}^{-1}\right)+1.717(\mathrm{r}=0.997)$. The linear curve indicated that the electrochemical reaction was predominantly adsorption-controlled in a confined thin film.

Analytical characteristics. A series of 4-NP solutions with different concentrations was measured using the optimized conditions. As a result, the peak current was linear to the 4-NP concentration over the range of $8.0 \times 10^{-8}$ to $4.0 \times 10^{-5} \mathrm{~mol} \mathrm{~L}^{-1}$. Fig. 7 shows the calibration curve of the sensor for the determination of 4-NP. The linear regression equation is $\operatorname{Ip}(10 \mu \mathrm{A})=$ $0.239 \mathrm{C}\left(\mu \mathrm{mol} \mathrm{L}{ }^{-1}\right)+0.988(\mathrm{r}=0.999)$. The detection limit was estimated to be $3.0 \times 10^{-8} \mathrm{~mol} \mathrm{~L}^{-1}$.

To explore the reproducibility of the sensor, a $4.0 \times 10^{-6} \mathrm{~mol}$ $\mathrm{L}^{-1}$ 4-NP solution was measured ten times, and the relative standard deviation of the peak current was $2.03 \%$. The $4-\mathrm{NP}$ solution was also determined with six electrodes that were fabricated independently, and the relative standard deviation of
Table 1. Analytical results of water samples and recovery test

\begin{tabular}{cccc}
\hline Sample & $\begin{array}{c}\text { Spiked } \\
\left(\mu \mathrm{mol} \mathrm{L}^{-1}\right)\end{array}$ & $\begin{array}{c}\text { Found } \\
\left(\mu \mathrm{mol} \mathrm{L}^{-1}\right)\end{array}$ & $\begin{array}{c}\text { Recovery } \\
(\%)\end{array}$ \\
\hline 1 & 0 & 0 & 0 \\
\hline \multirow{2}{*}{2} & 0.4 & 0.398 & 99.5 \\
& & 0.388 & 97.0 \\
3 & & 0.376 & 94.0 \\
\hline \multirow{3}{*}{4} & & 0.983 & 98.3 \\
& & 0.995 & 99.5 \\
& & 1.041 & 104.1 \\
& 2 & 2.042 & 102.1 \\
& & 2.008 & 100.4 \\
& & 2.101 & 105.0 \\
\hline
\end{tabular}

Table 2. Analytical results of apple samples and recovery test

\begin{tabular}{|c|c|c|c|}
\hline Sample & 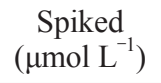 & $\begin{array}{l}\text { Found } \\
\left.(\mu \mathrm{mol} \mathrm{L})^{-1}\right)\end{array}$ & $\begin{array}{c}\text { Recovery } \\
(\%)\end{array}$ \\
\hline 1 & 0 & 0 & 0 \\
\hline \multirow{3}{*}{2} & \multirow{3}{*}{0.4} & 0.415 & 103.7 \\
\hline & & 0.408 & 102.0 \\
\hline & & 0.397 & 99.2 \\
\hline \multirow{3}{*}{3} & \multirow{3}{*}{1} & 1.043 & 104.3 \\
\hline & & 1.015 & 101.5 \\
\hline & & 0.974 & 97.4 \\
\hline \multirow{3}{*}{4} & \multirow{3}{*}{2} & 2.142 & 107.1 \\
\hline & & 2.018 & 100.9 \\
\hline & & 1.891 & 94.5 \\
\hline
\end{tabular}

the peak current was $3.14 \%$. Hence, the reproducibility was acceptable. The stability of the sensor was examined in PBS containing $4.0 \times 10^{-6} \mathrm{~mol} \mathrm{~L}^{-1} 4-\mathrm{NP}$ by means of voltammetry. The sensor was used and stored in redistilled water at room temperature for at least 2 weeks. The test results showed that the current responses of the sensor only had a deviation of $3.54 \%$ from its original current. The sensor therefore exhibited longtime stability.

The interference of some coexistent ions and molecules was examined. The results showed that for $4.0 \times 10^{-6} \mathrm{~mol} \mathrm{~L}^{-1}$ 4-NP, at least 100-fold concentration of $\mathrm{Ca}^{2+}, \mathrm{Mg}^{2+}, \mathrm{Cu}^{2+}, \mathrm{Mn}^{2+}$, $\mathrm{Pb}^{2+}, \mathrm{Zn}^{2+}, \mathrm{Fe}^{2+}, \mathrm{Fe}^{3+}$, and $\mathrm{Al}^{3+}$ and 10 -fold concentration of dopamine, ascorbic acid, vitamin $\mathrm{B}$, glucose, hypoxanthine, and cholesterol did not interfere with the determination of 4-NP. However, $o$-nitrophenol, $m$-nitrophenol, $p$-nitrobenzoic acid, and nitrobenzene severely interfered with the determination.

Determination of 4-NP in water and fruit samples. The sensor was used to determine 4-NP in natural water and fruit samples. A standard addition method was adopted to estimate the accuracy. The measurement results are shown in Tables 1 and 2. In addition, Fig. 8 shows the voltammograms from typical analysis of 4-NP in apple samples. No voltammetric peaks corresponding to 4-NP were observed when the samples were analyzed. This means that their concentrations were below the de- 


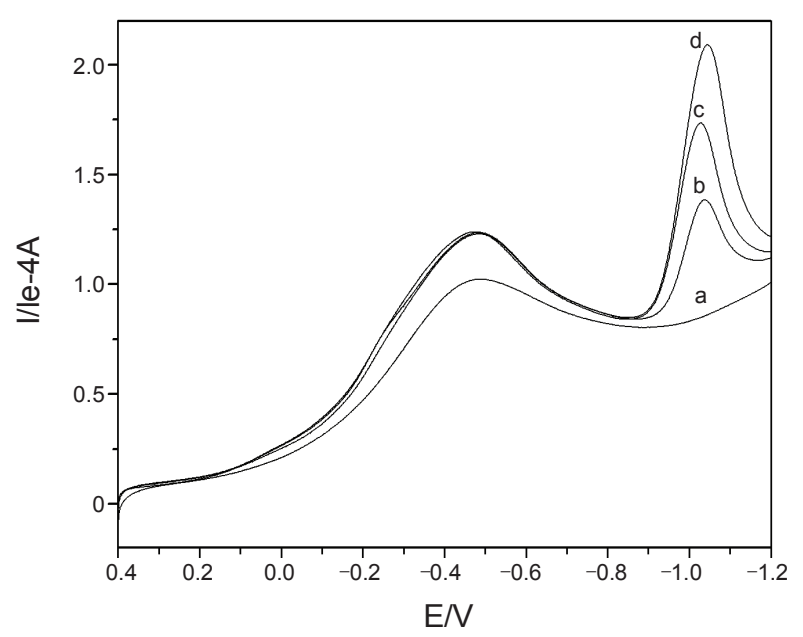

Figure 8. Voltammograms from typical analysis of 4-NP in apple samples. Curve a represents a apple sample without 4-NP spiked. Curve b, $\mathrm{c}$ and $\mathrm{d}$ represent the voltammograms of apple samples spiked with $4.0 \times 10^{-7}, 1.0 \times 10^{-6}, 2.0 \times 10^{-6} \mathrm{~mol} \mathrm{~L}^{-1} 4-\mathrm{NP}$ respectively.

tection limit. The recoveries of the standards added were close to $100 \%$, indicating that the method was reliable.

\section{Conclusions}

An electrochemical sensor for the determination of 4-NP has been developed based on the electropolymerization of safranine on GCE. The results suggest that the current response of the sensor to 4-NP is highly sensitive, selective and stable. The successful determination of 4-NP spiked into natural water and apple samples suggests that it is a promising electrochemical sensor for the detection of 4-NP in real samples.

\section{References}

1. Frenzel, W.; Frenzel, J. O.; Moeler, J. Anal. Chim. Acta 2006, 261,
253.

2. Penalver, A.; Pocurull, E.; Borrull, F.; Marce, R. M. J. Chromatogr. A 2002, 953, 79.

3. Nasr, B.; Abedllatif, G. J. Electrochem. Soc. 2005, 152(6), D113.

4. Cong, Y.; Wu, Z.; Ye, Q.; Tan, T. J. Zhejiang. SCI 2005, 2, 180.

5. Castillo, M.; Domingues, R.; Alpendurada, M. F.; Barcelo, D. Anal. Chim. Acta 1997, 353, 133.

6. Dzyadevych, S. V.; Chovelon J. M. Mater. Sci. Eng. C 2002, 21, 55.

7. ATSDR. Tox FAQs nitrophenols; Agency for Toxic Substances and Disease Registry, 2001.

8. Wang, S. P.; Chen, H. J. J. Chromatogr. A 2002, 979, 439.

9. Kontsas, H.; Rosenberg, C.; Pfaeffli, P.; Jappinen, P. Analysy. 1995, 120, 1745.

10. Brega, A.; Prandini, P.; Amaglio, C.; Pafuni, E. J. Chromatogr. A 1990, 535,313 .

11. Makuch, B.; Gazda, K. J. Chromatogr. A 1996, 733, 171.

12. Wissiack, R.; Rosenberg, E. J. Chromatogr. A 2002, 963, 149.

13. Kaniansky, D.; Krcmova, E.; Madajova, V.; Masar, M.; Marek, J. J. Chromatogr. A 1997, 772, 327.

14. Luz, R. D. S.; Damos, F. S.; De Oliveira, A. B.; Beek, J. Talanta 2004, 64, 935 .

15. Hu, S. S.; Xu, C. L.; Wang, G. P.; Cui, D. F. Talanta 2001, 54, 115.

16. Cordero-Rando, M. D.; Barea-Zamora, M.; Barber-Salvador, J. M.; Naranjo-Salvador, I. Microchim. Acta 1999, 132, 7.

17. Barek, J.; Ebertova, H.; Mejstrik, V.; Zima, J. Collect. Czech. Chem. C 1994, 59, 1761.

18. Ni, Y.; Wang, L.; Kokot, S. Anal. Chim. Acta 2001, 431, 101.

19. Lawrence, N. S.; Pagels, M.; Meredith, A.; Jones, T. G.. J.; Hall, C. E.; Pickles, C. S. J.; Godfried, H. P. Talanta 2006, 69, 829.

20. Lund, H., Baizer, M. M., Eds., Organic Electrochemistry: An Introduction and a Guide; Marcel Dekker: New York, 1991; 411.

21. Pedrosa, V. A.; Codognoto, L.; Avaca, L. A. J. Braz. Chem. Soc. 2003, 14, 530.

22. Pedrosa, V. A.; Codognoto, L.; Machado, S. A. S.; Avaca, L. A. J. Electroanal. Chem. 2004, 573, 11.

23. Garbellini, G. S.; Salazar-Banda, G. R.; Avaca, L. A. J. Braz. Chem. Soc. 2007, 18, 1095.

24. Ensafi, A. A. Anal. Lett. 2003, 36, 591.

25. Wang, B.; Dong, S. Talanta 2000, 51, 565.

26. Liu, X. Y.; Li, C. Y.; Hu, S. S. Microchim. Acta 2006, 154, 275.

27. Zhou, S.; Zhao, L. W.; Luo, L. N. Environ. Chem. 2006, 25, 683. 